\title{
EXAMPLE OF A POTENTIAL IN ONE-DIMENSIONAL SCATTERING PROBLEM FOR WHICH THERE ARE INFINITELY MANY PURELY IMAGINARY RESONANCES
}

\author{
A.G. RAMM \\ Mathematics Department, Kansas State University, Manhattan, KS 66506, USA
}

and

B.A. TAYLOR

Mathematics Department. University of Michigan, Ann Arbor, MI 48109, USA

Received 20 April 1987; accepted for publication 8 July 1987

Communicated by D.D. Holm

The inverse scattering theory methods are used to construct a real-valued potential $q(r) \in \mathrm{C}_{0}^{\infty}([0, R])$, where $R>0$ is an arbitrary fixed number, which gives rise to infinitely many purely imaginary resonances. This answers a question which has been open since the fifties.

\section{Introduction}

Consider the problem

$$
f^{\prime \prime}+k^{2} f-q(r) f=0, \quad r \geqslant 0, \quad q(r)=0 \quad \text { if } \quad r \geqslant R>0, \quad f \sim \exp (\mathrm{i} k r), \quad r \rightarrow \infty .
$$

If $q(r) \in \mathrm{L}^{2}$ problem (1) is uniquely solvable, the function $f(0, k):=f(k)$, the Jost function, is an entire funtion of $k$ of exponential type $\leqslant 2 R$, and $f(k)-1 \in \mathrm{L}^{2}(-\infty, \infty)$. The zeros of $f(k)$ in the half-plane $\operatorname{Im} k<0$ are called resonances. They are complex poles of the analytic continuation of the kernel of the operator [ $\left.-\mathrm{d}^{2} / \mathrm{d} r^{2}+q(r)-k^{2}\right]^{-1}$ in the spectral parameter $k$. Details can be found in ref. [1]. In this paper we deal with the one-dimensional problem (1) only. The known potentials give rise to a finite number of purely imaginary resonances [1]. It has been proved (see ref. [1] for a proof and references) that if $q(r) \approx c(R-r)^{\sigma}$ as $r \rightarrow R$, where $c$ and $\sigma$ are constants, $\sigma>0$, then there are at most finitely many purely imaginary resonances. The question of the existence of a potential which gives rise to infinitely many purely imaginary resonances in the one-dimensional case has been open since the fifties. In ref. [2] it was proved that in the three-dimensional problem there exist infinitely many purely imaginary resonances provided that $q(\kappa)$ is compactly supported and does not change sign. This result does not answer the open question in the one-dimensional case because in the one-dimensional case the potentials which do not change sign and are compactly supported give rise to finitely many purely imaginary resonances. In ref. [3] it was proved that if $f(k)$ is an entire function of exponential type, $f(k)-1 \in \mathrm{L}^{2}(-\infty$, be), and $f(k)$ is a Jost function for an underlying potential $q(x)$ then $q(x)$ is compactly supported. In ref. [4] an idea from ref. [3] was used in a new proof of the existence of infinitely many resonances (not necessarily purely imaginary). This proof is based on the inverse scattering theory. The purpose of this paper is to give an example of a real-valued potential, with support in $[0, R]$, which gives rise to infinitely many purely imaginary resonances. Here $R>0$ is an arbitrary fixed number. The potential we construct is in $\mathrm{C}_{0}^{\infty}([0, R])$. 


\section{The construction}

It is well known $[1,5]$ that the solution to (1) can be written as

$$
f(r, k)=\exp (\mathrm{i} k r)+\int_{r}^{\infty} A(r, t) \exp (\mathrm{i} k t) \mathrm{d} t
$$

so

$$
f(k)=1+\int_{0}^{\infty} A(t) \exp (\mathrm{i} k t) \mathrm{d} t, \quad A(t):=A(0, t) .
$$

The function $A(r, t)$ solves the Marchenko equation

$$
A(r, t)+F(r+t)+\int_{r}^{\infty} A(r, y) F(y+t) \mathrm{d} y=0, \quad t \geqslant r,
$$

$A(r, t)=0$ if $t<r$. The function

$$
F(t):=\frac{1}{2 \pi} \int_{-\infty}^{\infty} \mathrm{d} k \exp (\mathrm{i} k t)[1-S(k)]
$$

where $S(k):=f(-k) / f(k)$ is the $S$-matrix and we assumed that $q(r)$ does not produce bound states. If $q(r)=0$ for $r \geqslant R$ then the estimate (1.3.3) in ref. [5], p. 20, shows that $A(r, t)=0$ for $r+t \geqslant 2 R$. Therefore $A(t)=0$ if $t \geqslant 2 R$. Thus we have

Lemma 1. If $q(r)=0$ for $r \geqslant R$ then $f(k)=1+\int_{0}^{2 R} A(t) \exp (\mathrm{i} k t) \mathrm{d} t$.

Corollary 1. If $q(r)=0$ for $r \geqslant R, q(r) \in \mathrm{L}^{2}$, then $f(k)$ is an entire function of exponential type $\leqslant 2 R$.

\section{Sketch of the ideas}

In order to construct $q(r)$ which gives rise to infinitely many purely imaginary resonances we construct a real-valued function $A(t) \in \mathrm{C}_{0}^{\infty}([0,2 R])$ such that:

(i) $f\left(-\mathrm{i} b_{j}\right)=0, b_{j}>0, b_{j} \rightarrow \infty$ and,

(ii) $|f(k)|>0$ if $\operatorname{Im} k \geqslant 0$.

If $A(t)$ is constructed then $q(r)$ can be obtained via the inverse scattering methods from $f(k)$ given by $(2)$. For example, given $f(k)$ one constructs $S(k):=f(-k) / f(k)$ then $F(t)$ by formula (4), then $A(r, t)$ by solving eq. (3), and then $q(r)$ by the well-known formula $q(r)=-2 \mathrm{~d} A(r, r) / \mathrm{d} r$. We will prove that the function $A(t)$ which we construct below in lemma 2 produces the function $S(k)$ which satisfies the necessary conditions for this function to be an $S$ matrix corresponding to a potential $q(r)$ which produces no bound states. We then prove that $q(r) \in \mathrm{C}_{0}^{\infty}([0, R])$.

Lemma 2. There exists a real-valued function $A(t) \in \mathrm{C}_{0}^{\infty}(0, R)$ such that $f(k):=1+\int_{0}^{2 R} A(t) \exp (\mathrm{i} k t) \mathrm{d} t$ has infinitely many purely imaginary zeros $k_{j}=-\mathrm{i} b_{j}, b_{j} \rightarrow \infty, 0<b_{1}<b_{2}<b_{3}<\ldots$ and no zeros in $\operatorname{Im} k \geqslant 0$. The number $b_{1}>0$ can be made arbitrarily large, the function $A(t)$ can be chosen real-valued, the number $R>0$ is an arbitrary fixed number. 
Proof. Let $\phi(b):=1+\int_{0}^{2 R} A(t) \exp (b t) \mathrm{d} t$; let $\left\{A_{j}\right\}$ be an infinite set of intervals $\left(c_{j}, c_{j}+h_{j}\right), j=1,2, \ldots$, $0<c_{j}<c_{j}+h_{j}<c_{j+1}<2 R, c_{j} \rightarrow R^{\prime}<2 R$ as $j \rightarrow \infty$; let $\left\{\epsilon_{j}\right\}_{j=0}^{\infty}$ be a decreasing sequence of positive numbers which will be defined inductively in the course of the proof; let $0 \leqslant \eta_{j} \in \mathrm{C}_{0}^{\infty}\left(\Delta_{j}\right)$ be a sequence of functions such that $\int \eta_{j} \mathrm{~d} t=1, j=1,2,3, \ldots, \int:=\int_{0}^{2 R}$, and let $\psi_{j}(b):=\int \eta_{j} \exp (b t) \mathrm{d} t$. Let us choose a decreasing sequence $\epsilon_{j} ; \epsilon_{0}>\epsilon_{1}>\epsilon_{2}$ $>\ldots>0$, so that the series $A(t)=\sum_{j=1}^{\infty}(-1)^{j+1} \epsilon_{j} \eta_{j}(t)$ converges, and choose $b_{j}^{\prime}<b_{j+1}^{\prime} \rightarrow \infty$ so that:

(i) $A(t) \in \mathrm{C}_{0}^{\infty}([0, R])$, and

(ii) $\phi\left(b_{j}^{\prime}\right)>0, \phi\left(b_{j+1}^{\prime}\right)<0$, where $b_{j}^{\prime}<b_{j}<b_{j+1}^{\prime}<b_{j+1}, b_{j}^{\prime} \rightarrow \infty$, and $\phi\left(b_{j}\right)=0$.

In the following argument we can make $\epsilon_{j}$ even smaller if needed. Let us show by induction that the desired choice of $\epsilon_{j}$ and $b_{j}^{\prime}$ is possible. Clearly, if $\epsilon_{j} \rightarrow 0$ sufficiently fast then $A(t) \in \mathrm{C}_{0}^{\infty}([0, R])$. Let $b_{1}^{\prime}>0$ be arbitrary. Then $1+\epsilon_{1} \psi_{1}\left(b_{1}^{\prime}\right)>1>0$. Since $\psi_{j+1}(b) \psi_{j}^{-1}(b) \rightarrow \infty$ as $b \rightarrow \infty$ one can choose $b_{2}^{\prime}>b_{1}^{\prime}$ and $\epsilon_{2}<\epsilon_{1}$ such that $\phi_{2}\left(b_{1}^{\prime}\right):=1+\epsilon_{1} \psi_{1}\left(b_{1}^{\prime}\right)-\epsilon_{2} \psi_{2}\left(b_{1}^{\prime}\right)>1 ; \phi_{2}\left(b_{2}^{\prime}\right):=1+\epsilon_{1} \psi_{1}\left(b_{2}^{\prime}\right)-\epsilon_{2} \psi_{2}\left(b_{2}^{\prime}\right)<-1$.

Having so chosen $\epsilon_{2}$ and $b_{2}^{\prime}$, choose $\epsilon_{3}$ and $b_{3}^{\prime}$ so that

$$
\phi_{3}\left(b_{1}^{\prime}\right):=1+\epsilon_{1} \psi_{1}\left(b_{1}^{\prime}\right)-\epsilon_{2} \psi_{2}\left(b_{1}^{\prime}\right)+\epsilon_{3} \psi_{3}\left(b_{1}^{\prime}\right)>1, \quad \phi_{3}\left(b_{2}^{\prime}\right)<-1, \quad \phi_{3}\left(b_{3}^{\prime}\right)>1 .
$$

Continue in this fashion to obtain

$$
\phi_{n}\left(b_{j}^{\prime}\right)>1, \quad j=1,3,5, \ldots, \quad j \leqslant n, \quad \phi_{n}\left(b_{j}^{\prime}\right)<-1, \quad j=2,4,6, \ldots, \quad j \leqslant n,
$$

where

$$
\phi_{n}(b):=1+\sum_{j=1}^{n}(-1)^{j+1} \epsilon_{j} \psi_{j}(b), \quad \phi_{n}(b) \rightarrow \phi(b) \quad \text { as } n \rightarrow \infty
$$

Passing to the limit $n \rightarrow \infty$ in (5) yields

$$
\phi\left(b_{j}^{\prime}\right)>1, \quad j=1,3,5, \ldots, \quad \phi\left(b_{j}^{\prime}\right)<-1, \quad j=2,4,6, \ldots .
$$

Since $\phi(b)$ is continuous and real valued, (6) implies that there exists a sequence $b_{j} \rightarrow \infty, b_{j}^{\prime}<b_{j}<b_{j+1}^{\prime}$, such that $\phi\left(b_{j}\right)=0, j=1,2, \ldots$. By choosing $\epsilon_{1}$ sufficiently small one can obtain $f(k)$ such that $|\mathrm{f}(k)| \geqslant \frac{1}{2}$ in the halfplane $\operatorname{Im} k \geqslant 0$. Lemma 2 is proved.

Theorem 1. There exists a real-valued potential $q(r) \in \mathrm{C}_{0}^{\infty}([0, R])$, where $R>0$ is an arbitrary fixed number, which gives rise to infinitely many purely imaginary resonances $k_{j}=-\mathrm{i} b_{j}, b_{j}>0, b_{j} \rightarrow \infty$ as $j \rightarrow \infty$.

Proof. Let $f(k)$ be defined by (2) where $A(t)$ is constructed in lemma 2. Define $S(k)=f(-k) / f(k)$. Note that: $S(k)$ is meromorphic on the whole complex $k$-plane $\mathrm{C}$; analytic in $\mathrm{C}_{+}=\{k: \operatorname{Im} k \geqslant 0\} ;|S(k)|=1$ if $-\infty<k<\infty$ since $A(t)$ is real-valued; $S(k)-1$ decays faster than $|k|^{-a}$ as $k \rightarrow \pm \infty$ for any $a>0$ since $A(t) \in \mathrm{C}_{0}^{\infty}$; the function $F(t):=(2 \pi)^{-1} \int_{-\infty}^{\infty}[1-S(k)] \exp (\mathrm{i} k t) \mathrm{d} k$ is real-valued since $\overline{S(k)}=S(-k)$, where the bar denotes complex conjugate; the function $F(t) \in \mathrm{C}^{\infty}(-\infty, \infty)$ and $\left(1+t^{2}\right)^{a} F^{(n)}(t) \in \mathrm{L}^{2}(-\infty, \infty)$ for any $a>0$ and $n>0$, since $1-S(k)$ has these properties as a function of $k,-\infty<k<\infty$.

In ref. [5], p. 105, it is proved that a function $S(k)$ is the $S$-matrix corresponding to a real-valued potential $q(r)$ such that $\int_{0}^{\infty}(1+r)|q(r)| \mathrm{d} r<\infty$ and $q$ produces no bound states iff the following conditions are satisfied: (1) $F(t) \in \mathrm{L}^{1}$ is a real-valued function, $\int_{0}^{\infty} t\left|F^{\prime}(t)\right| \mathrm{d} t<\infty$, (2) the equations

$$
-h_{1}(t)+\int_{-\infty}^{0} h_{1}(r) F(t+r) \mathrm{d} r=0, \quad-\infty<t \leqslant 0,
$$




$$
h(t)+\int_{0}^{\infty} h(r) F(t+r) \mathrm{d} r=0, \quad 0 \leqslant t<\infty
$$

have only the trivial solutions $h_{1}=0$ in $\mathrm{L}^{1}(-\infty, 0)$ and $h=0$ in $\mathrm{L}^{1}(0, \infty)$. Clearly condition (1) is satisfied by our $F(t)$. Let us check that condition (2) is satisfied as well. Let $h \in \mathrm{L}^{1}(0, \infty)$ solve (8). Since $F$ is realvalued we can assume that $h$ is real-valued, $h=0$ for $t<0$. Multiply ( 8$)$ by $h$ and integrate over $(0, \infty)$ to get

$$
\begin{aligned}
0 & =\int_{0}^{\infty} h^{2} \mathrm{~d} t+\int_{0}^{\infty} \int_{0}^{\infty} \mathrm{d} r \mathrm{~d} t h(r) h(t) \frac{1}{2 \pi} \int_{-\infty}^{\infty}[1-S(k)] \exp [\mathrm{i} k(r+t)] \mathrm{d} k \\
& =\int_{0}^{\infty} h^{2} \mathrm{~d} t+\frac{1}{2 \pi} \int_{-\infty}^{\infty} \tilde{h}^{2}(k)[1-S(k)] \mathrm{d} k,
\end{aligned}
$$

where $\tilde{h}(k)=\int_{0}^{\infty} h(t) \exp (\mathrm{i} k t) \mathrm{d} t$. Note that $\bar{h}(k)=\tilde{h}(-k)$,

$$
\int_{0}^{\infty} h^{2} \mathrm{~d} t=\frac{1}{2 \pi} \int_{-\infty}^{\infty} \tilde{h}(k) \tilde{h}(-k) \mathrm{d} k \text {, and } \frac{1}{2 \pi} \int_{-\infty}^{\infty} \tilde{h}^{2}(k) \mathrm{d} k=\int_{-\infty}^{\infty} h(r) h(-r) \mathrm{d} r=0
$$

since $h(r)=0$ for $r<0$. Thus $0=\int_{-\infty}^{\infty}|\tilde{h}|^{2} \mathrm{~d} k-\int_{-\infty}^{\infty} S(k) \tilde{h}^{2}(k) \mathrm{d} k$. Let

$$
\|\tilde{h}\|^{2}=\int_{-\infty}^{\infty}|\tilde{h}|^{2} \mathrm{~d} k, \quad \int_{-\infty}^{\infty} S \tilde{h}^{2} \mathrm{~d} k=\int_{-\infty}^{\infty} S \tilde{h} \cdot \overline{\bar{h}(-k)} \mathrm{d} k:=(S \tilde{h}, \tilde{h}(-k)) .
$$

Since $|S(k)|=1$ one has $(S \tilde{h}, \tilde{h}(-k)) \leqslant\|\tilde{h}\|^{2}$, and the equality is possible only if $S \tilde{h}=\tilde{h}(-k)$. We saw above that $\|\tilde{h}\|^{2}=(S \tilde{h}, \tilde{h}(-k))$. Therefore, $S \tilde{h}=\tilde{h}(-k)$. This can be written as

$$
\tilde{h}(k) / f(k)=\tilde{h}(-k) / f(-k)
$$

by the definition of $S(k)$. The left-hand side of $(9)$ is analytic in $\mathrm{C}_{+}$, the right-hand side of (9) is analytic in $\mathrm{C}_{-}:=\mathrm{C} \backslash \mathrm{C}_{+}$. Since $h(k) \rightarrow 0$ as $|k| \rightarrow \infty, \operatorname{Im} k \geqslant 0$, and $f(k) \rightarrow 1$ as $|k| \rightarrow \infty, \operatorname{Im} k \geqslant 0$, one concludes that the function $\tilde{h}(k) / f(k)$ is analytic in $C$ and vanishes at infinity. Therefore $\tilde{h}=0$ and $h=0$. We proved that eq. (8) has only the trivial solution in $L^{1}(0, \infty)$.

Let us prove that eq. (7) has only the trivial solution in $\mathrm{L}^{1}(-\infty, 0)$. Let $h_{1}$ solve (7). We can assume that $h_{1}$ is real-valued, $h_{1}=0$ for $t>0$. Let $t=-\tau, r=-\rho, h_{1}(-t)=g(t)$. Then (7) becomes

$$
-g(\tau)+\int_{0}^{\infty} g(\rho) F(-\tau-\rho) \mathrm{d} \rho=0, \quad \tau \geqslant 0, \quad g(\tau)=0 \quad \text { if } \quad \tau \leqslant 0 .
$$

By the argument similar to the one given above we conclude from (10) that

$$
\int_{-\infty}^{\infty}|\tilde{g}|^{2} \mathrm{~d} k=\frac{1}{2 \pi} \int_{-\infty}^{\infty} \tilde{g}^{2}(-k)[1-S(k)] \mathrm{d} k=-\frac{1}{2 \pi} \int_{-\infty}^{\infty} \tilde{g}^{2}(-k) S(k) \mathrm{d} k,
$$

that $\tilde{g}(k)=S(k) \tilde{g}(-k)$, and that $\tilde{g}(k) f(k)=f(-k) \tilde{g}(-k)$. The last equation and the analytic properties of $f(k)$ and $\tilde{g}(k)$ imply that $\tilde{g} f(k)=0$. Therefore $\tilde{g}=0$ and $g(\tau)=0$. Thus $h_{1}=0$, and we proved that eq. (7) has only the trivial solution.

Therefore $S(k):=f(-k) / f(k)$, where $f(k)$ is defined by (2) and $A(t)$ is constructed in lemma 2 , is the $S$ matrix corresponding to the potential $q(r)$ which can be obtained by the formula $q(r)=-2 \mathrm{~d} A(r, r) / \mathrm{d} r$. In this 
formula the function $A(r, t)$ is the unique solution of the Marchenko equation (3) in which $F(t)$ is given by (4).

To finish the proof of theorem 1 we have to prove that $q(r)=-2 \mathrm{~d} A(r, r) / \mathrm{d} r \in \mathrm{C}_{0}^{\infty}([0, R])$. In the proof the method given in ref. [3] will be used. Note, that the fact that $q(r) \in \mathrm{C}^{\infty}$ has been already established implicitly. Indeed, we proved that $F(t) \in \mathrm{C}^{\infty}$ and $\left(1+t^{2}\right)^{a} F^{(n)}(t) \in \mathrm{L}^{2}(-\infty, \infty)$ for any $a>0$ and $n>0$. This and eq. (3) which is uniquely solvable as we have proved) imply that $A(r, t)$ is infinitely differentiable in its arguments. So, we have to prove that $q(r)=0$ for $r \geqslant R$. Eq. (3) for $r=t$ takes the form

$$
A(r, r)+F(2 r)+\int_{r}^{\infty} A(r, y) F(y+r) \mathrm{d} y=0 .
$$

It follows from (11) that $q(r)=0$ for $r \geqslant R$ provided that

$$
F(t)=0 \text { for } t \geqslant 2 R \text {. }
$$

To prove (12) we use eq. (3) with $r=0$ :

$$
A(t)+F(t)+\int_{0}^{\infty} A(y) F(y+t) \mathrm{d} y=0, \quad t \geqslant 0 .
$$

The function $A(t)=0$ for $t \geqslant 2 R$ according to lemma 2. Let us write (13) as

$$
A(t)+F(t)+\int_{t}^{\infty} A(v-t) F(v) \mathrm{d} v=0, \quad t \geqslant 0
$$

and consider (14) as a Volterra equation for $F(t)$. The kernel $A(v)=0$ if $v \leqslant 0$ or $v \geqslant 2 R$. Let us denote the lefthand side of (14) for $t<0$ by $G_{-}(t), G_{-}(t):=0$ for $t \geqslant 0$. Then

$$
A(t)+F(t)+\int_{t}^{\infty} A(v-t) F(v) \mathrm{d} v=G_{-}(t), \quad-\infty<t<\infty .
$$

Since $A(t)=0$ for $t<0$, one can extend the integration in (15) to $-\infty$. Take the Fourier transform of (15) to get

$$
\tilde{A}(\lambda)+\tilde{F}(\lambda)+\tilde{F}(\lambda) \tilde{A}(-\lambda)=\tilde{G}_{-}(\lambda) \text { where } \tilde{A}(\lambda):=\int_{-\infty}^{\infty} A(t) \exp (\mathrm{i} \lambda t) \mathrm{d} t .
$$

Thus

$$
\tilde{F}=\left[\tilde{G}_{-}(\lambda)-\tilde{A}(\lambda)\right] /[1+\tilde{A}(-\lambda)], \quad-\infty<\lambda<\infty .
$$

We can assume that $1+\tilde{A}(-\lambda) \neq 0, \operatorname{Im} . \lambda \leqslant 0$, since $f(k) \neq 0$ if $\operatorname{Im} k \geqslant 0$. Therefore $[1+\tilde{A}(-\lambda)]^{-1}=1+\widetilde{B}(-\lambda)$, where $\widetilde{B}(\lambda)=\int_{0}^{\infty} \exp (-\mathrm{i} \lambda t) B(t) \mathrm{d} t$ and $B(t)$ is a smooth function. Thus (17) is of the form

$$
\tilde{F}=\tilde{G}_{-}(\lambda)\left[1+\tilde{B}_{-}(\lambda)\right]-\tilde{A}\left[1+\tilde{B}_{-}(\lambda)\right], \text { where } \tilde{B}_{-}(\lambda)=\tilde{B}(\lambda)=\int_{-\infty}^{0} \mathrm{e}^{\mathrm{i} \lambda t} B(-t) \mathrm{d} t .
$$

It is now easy to see from (18) that 


$$
\int_{-\infty}^{\infty}|\tilde{F}(\lambda-\mathrm{i} \mu)|^{2} \mathrm{~d} \lambda \leqslant c \exp (4 R \mu), \quad \mu>0 .
$$

By a theorem of Titchmarsh (ref. [6], theorem 96), (19) implies that $F(t)=0$ for $t>2 R$. Theorem 1 is proved. Remark 1. The Titchmarsh theorem can be stated as follows: iff $F(t)=0$ for $t<-R$, the Fourier transform $\tilde{F}(\lambda)=\int_{-\infty}^{\infty} F(t) \exp (\mathrm{i} \lambda t) \mathrm{d} t$ satisfies the inequality $\int_{-\infty}^{\infty}|\tilde{F}(\lambda+\mathrm{i} \mu)|^{2} \mathrm{~d} \lambda \leqslant c \exp (2 R \mu), \mu>0, c=$ const.

This can be restated as: iff $F(t)=0$ for $t>R$ the inequality $\int_{-\infty}^{\infty}|\tilde{F}(\lambda-\mathrm{i} \mu)|^{2} \mathrm{~d} \lambda \leqslant c \exp (2 R \mu), \mu>0, c=$ const, holds. This last statement was used in connection with inequality (19).

Remark 2. Our argument also proves the fact first established in ref. [3]: if $f(k)$ is an entirc function of exponential type and $f(k)-1 \in \mathrm{L}^{2}(-\infty, \infty)$ the potential $q(x)$ is compactly supported. The converse statement is well-known and easy to prove: if $q(r)$ is compactly supported and $\int_{0}^{\infty}(1+r)|q(r)| \mathrm{d} r<\infty$, then $f(k)-1 \in$ $\mathrm{L}^{2}(-\infty, \infty)$ and $f(k)$ is an entire function of exponential type [1].

Remark 3. We have chosen the way to reconstruct $q(r)$ from the knowledge of $f(k)$ via the Marchenko equation. The other way is via the Gelfand-Levitan (G-L) equation. If $f(k)$ is known and $f(k)$ does not have zeros in $\mathrm{C}_{+}$, then the spectral function $\mathrm{d} \rho=(2 / \pi) k^{2} \mathrm{~d} k /|f(k)|^{2}$ for $k>0, \mathrm{~d} \rho=0$ for $k<0$, and one can find $q(r)$ given $\mathrm{d} \rho$ by the formula $q(r)=2 \mathrm{~d} K(r, r) / \mathrm{d} r$, where $K(r, t)$ is the (unique) solution of the G-L equation:

$$
K(r, t)+\int_{0}^{r} K(r, y) L(y, t) \mathrm{d} y+L(r, t)=0, \quad 0 \leqslant t<r,
$$

where the kernel $L(r, t)$ is defined by the formula

$$
L(r, t)=\int_{0}^{\infty} \frac{\sin k t}{k} \frac{\sin k r}{k} \mathrm{~d}\left(\rho-\rho_{0}\right), \quad \mathrm{d}\left(\rho-\rho_{0}\right)=\frac{2}{\pi} k^{2}\left(|f|^{-2}-1\right) \mathrm{d} k .
$$

Remark 4. From the inequality $|f(k)| \leqslant 1+\sum_{n=0}^{\infty}|k|^{n} A_{n} / n !$, where $A_{n}:=\int_{0}^{\infty}|A(0, t)| t^{n} \mathrm{~d} t$, one can see that $f(k)$ is an entire function of a finite order $\rho<\infty$, if $A_{n}=\mathrm{O}\left(n^{n a}\right)$ as $n \rightarrow \infty, a<1$. This follows from the formula (1.05) in ref. [7]:

$$
\rho=\lim _{n \rightarrow \infty} \sup \frac{n \ln n}{\ln \left(n ! A_{n}\right)}=\frac{1}{1-a}, \text { where } \quad a=\lim _{n \rightarrow \infty} \sup \frac{\ln A_{n}}{n \ln n}<1 .
$$

An entire function $f(k)$ of a finite order, bounded in $\mathrm{C}_{+}$, such that $f(k) \rightarrow 1$ as $|k| \rightarrow \infty, \operatorname{Im} k \geqslant 0$, and $f(k) \not \equiv 1$ has infinitely many zeros. This follows immediately from the Hadamard theorem ([7] $\$ 10$, theorem 13 ). Therefore, if $A_{n}=\mathrm{O}\left(n^{n a}\right), a<1$, and $f(k) \not \equiv 1$, then $f(k)$ has infinitely many zeros and the corresponding $q(r)$ produces infinitely many resonances (not necessarily purely imaginary).

One can write a sufficient condition for the inequality $a<1$ to hold in terms of $q(r)$. Namely, $A_{n} \leqslant$ $c \int_{0}^{\infty} \mathrm{d} t t^{n} \int_{t}^{\infty}|q| \mathrm{d} r=c(n+1)^{-1} \int_{0}^{\infty} t^{n+1}|q| \mathrm{d} t$. Therefore if $Q_{n}:=\int_{0}^{\infty} r^{n}|q(r)| \mathrm{d} r=\mathrm{O}\left(n^{n a}\right)$ as $n \rightarrow \infty$ for some $a<1$ then $A_{n}=\mathrm{O}\left(n^{n a}\right)$ as $n \rightarrow \infty$ with the same $a<1$. Therefore if $Q_{n}=\mathrm{O}\left(n^{n a}\right), a<1$, as $n \rightarrow \infty$, and $q(r) \not \equiv$ then $q(r)$ produces infinitely many resonances (not necessarily purely imaginary). This observation was made by J. Hwang after A.G. Ramm made paper [4] available to him.

\section{Bibliographical remarks}

An extensive bibliography on resonances for one-dimensional scattering problems can be found in refs. $[1,12]$ where the works of Humblet, Rollnik, Regge, Sartori, Nussenzweig and others are mentioned. The three-dimensional case is discussed in ref. [8], pp. 365-393, where a sharp bound on the location of the resonances is given; 
existence of infinitely many purely imaginary resonances is proved for $q(x)$ which does not change sign; methods for calculating resonances are given and their convergence is proved; connection between the resonances and the SEM (singularity expansion method) is established; and perturbation theory for resonances is discussed. Variational principles for resonances are known [9]. An extensive bibliography on resonances can be found in ref. [10]. A recent article is ref. [11], and some of its results (e.g. lemmas 8, 9 and part 1 of theorem 1) can be obtained by the method given in ref. [4] and in the present paper.

The condition $Q_{n}=\mathrm{O}\left(n^{n a}\right), a<1$, covers all the previously published sufficient conditions for existence of infinitely many resonances for one-dimensional radial Schrödinger operators as far as we know. The methods of refs. [3,4] are extended in refs. [13,14].

\section{Acknowledgement}

A.G. Ramm thanks O.L. Weaver for a discussion and useful comments and ONR for support.

\section{References}

[1] R. Newton, Scattering theory of waves and particles (Springer, Berlin, 1982).

[2] A.G. Ramm, Phys. Lett. A 101 (1984) 187.

[3] A.G. Ramm, Sov. Phys. Dokl. 157 (1964) 1073.

[4] A.G. Ramm, Existence of infinitely many resonances, preprint (1986).

[5] Z. Agranovich and V. Marchenko, The inverse problem of scattering theory (Gordon and Breach, New York, 1963).

[6] E. Titchmarsh, Theory of Fourier integrals (Oxford Univ. Press, Oxford, 1948).

[7] B. Levin, Distribution of zeros of entire functions, Am. Math. Soc. Transl., Vol. 5. (Am. Math. Soc., Providence, 1972).

[8] A.G. Ramm, Scattering by obstacles (Reidel, Dordrecht, 1986).

[9] A.G. Ramm, J. Math. Phys. 21 (1980) 2052; 23 (1982) 1112.

[10] S. Albeverio and R. Hoegh-Krohn, J. Math. Anal. Applic. 101 (1984) 491.

[11] E. Skibsted, J. Math. Anal. Appl. 117 (1986) 153.

[12] C. Chadan and P. Sabatier, Inverse problems in quantum scattering (Springer, Berlin, 1977).

[13] A.G. Ramm, Necessary and sufficient conditions on the scattering data for the potential to be in $L^{2}$ for the Schrödinger operator on the half-line, Inverse Problems 3 (1987), to be published.

[14] A.G. Ramm, Inverse scattering on half-line, submitted for publication. 